\title{
IMPURITY-RELATED TERAHERTZ EMISSION FROM QUANTUM WELL NANOSTRUCTURES
}

\author{
D.A. Firsov ${ }^{\text {a }}$, L.E. Vorobjev a, V.Yu. Panevin a , A.N. Sofronov ${ }^{\text {a }}$, R.M. Balagula ${ }^{\text {a }}$, \\ and D.V. Kozlov ${ }^{b}$ \\ ${ }^{a}$ St. Petersburg State Polytechnical University, Politechnicheskaya 29, 195251 St. Petersburg, Russia \\ E-mail:dmfir@rphf.spbstu.ru \\ ${ }^{\mathrm{b}}$ Institute for Physics of Microstructures, Russian Academy of Sciences, 603950 Nizhny Novgorod, Russia
}

Received 18 November 2013; accepted 4 December 2013

\begin{abstract}
Terahertz emission in GaAs/AlGaAs quantum well structures doped with shallow impurities was studied in conditions of interband optical excitation for n-doped structures and impurity breakdown in the lateral electric field for p-doped structures. Emission spectra were obtained. It was shown that the observed emission is related to optical transitions of charge carriers between impurity levels and to impurity-band transitions. The depopulation of the final states under interband optical pumping was realized with recombination of non-equilibrium holes and electrons localized at neutral donors.
\end{abstract}

Keywords: terahertz emission, impurity, quantum wells, optical excitation, impurity breakdown, GaAs/AlGaAs

PACS: 78.55.Cr , 78.60.Fi, 78.67.De, 78.70.Gq

\section{Introduction}

The energies of intracentre carrier transitions in doped semiconductors and semiconductor nanostructures correspond to terahertz spectral ranges. That is why the optical studies of impurity-related carrier transitions attract particular interest with respect to the development of the new types of terahertz radiation sources. In order to get terahertz radiation one should depopulate the final states. There are some ways for such depopulation. In Ref. [1] spontaneous emission in the $\mathrm{THz}$ range was observed from electrically pumped phosphorus-doped bulk silicon. Another way to create non-equilibrium carriers and depopulate final states of intracentre transitions can be realized with the impurity-band optical excitation by the radiation of the $\mathrm{CO}_{2}$ laser (see for example [2] where $\mathrm{THz}$ lasing was observed in bulk Si:P).

A convenient way to depopulate final states is the interband optical pumping. In Ref. [3] terahertz luminescence under the interband photoexcitation of bulk $\mathrm{n}-\mathrm{GaAs}$ and $\mathrm{p}-\mathrm{Ge}$ semiconductors at low temperatures was reported. The terahertz photoluminescence was caused by intraband radiative carrier transitions which accompany the capture of non-equilibrium carriers by impurity centres. The depopulation of impurity ground states was achieved due to the impurity-assisted electron-hole recombination.
Investigation of impurity-related $\mathrm{THz}$ emission in nanostructures with quantum wells $(\mathrm{QW})$ is also of considerable interest because in this case the energy distances between levels can be easily changed with changing the structure parameters. In Ref. [4] terahertz emission was observed in the $\mathrm{n}-\mathrm{GaAs} / \mathrm{AlGaAs}$ quantum well structure under impurity breakdown in a strong electric field. This emission was related to electron intraband optical transitions from the conduction band to donor levels, transitions between bound donor states, and transitions between resonant and bound donor states.

In this work, we present the results of the observations of terahertz emission in GaAs/AlGaAs-doped quantum well structures. We used two ways of the excitation of non-equilibrium charge carriers: interband optical pumping and lateral electric field. We connect the observed emission with optical transitions of charge carriers between impurity levels and with impurity-band transitions.

\section{THz emission under interband optical pumping}

In conditions of low-temperature interband optical excitation of n-doped QW nanostructures, the recombination of non-equilibrium holes and electrons localized at neutral donors can exist along with exciton 
recombination. We studied the terahertz radiation related to impurity-band and intracentre electron transitions accompanying the process of the capture of non-equilibrium electrons by charged donors.

Samples for $\mathrm{THz}$ studies were grown by molecular beam epitaxy (MBE) on a GaAs semi-insulating substrate and consisted of 50 layers of $30 \mathrm{~nm}$ GaAs quantum wells separated with $7 \mathrm{~nm} \mathrm{Al}_{0.3} \mathrm{Ga}_{0.7}$ As barriers. Quantum wells were doped in the $4 \mathrm{~nm}$ central region with the surface donor concentration of $3 \cdot 10^{10} \mathrm{~cm}^{-2}$.

Emission spectra were measured with the fourier transform infrared (FTIR) spectrometer Bruker Vertex 80v equipped with a liquid He cooled Si bolometer operating in a step-scan mode and lock-in amplifier SR830. The sample was mounted in a closed-cycle He cryostat Janis PTCM-4-7. Its temperature can be varied from 4.2 to $300 \mathrm{~K}$. A solid state CW laser with diode pumping $(\lambda=532 \mathrm{~nm}, P=8 \mathrm{~mW})$ was used for interband optical excitation. Laser current was modulated with the frequency of $87 \mathrm{~Hz}$.

The experimental spectrum obtained at $T=4.4 \mathrm{~K}$ is shown in Fig. 1. It should be noted that the real emission spectra can slightly differ from spectra presented in the present paper because of spectral dependence of photodetector sensitivity and beamsplitter transmission.

The emission spectrum consists of two discernible regions of 5-12 and 15-28 meV. Ionization energy of $\mathrm{Si}$ impurity in the investigated structure as it was calculated in Ref [ 4 ] is about $8.8 \mathrm{meV}$. It follows that the emission in the range of $15-28 \mathrm{meV}$ cannot be connected with Si-related electron transitions. We suggest the following explanation of the emission in this spectral range. It is based on the emission spectrum obtained in similar experimental conditions from the GaAs substrate without any QW layers. This spectrum does not contain features in the range of $5-12 \mathrm{meV}$ but essentially copies the spectrum shown in Fig. 1 in the

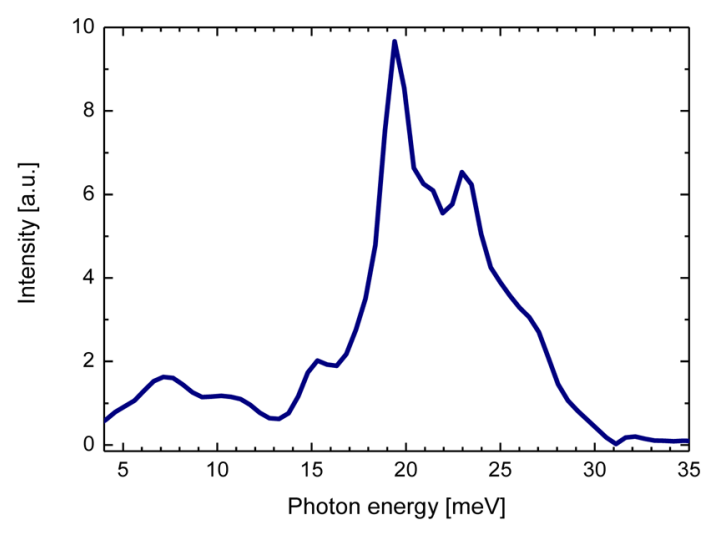

Fig. 1. Terahertz emission spectrum of the optically excited GaAs/GaAs QW structure at $T=4.4 \mathrm{~K}$. range of 15-28 meV. It is well known that the energies of intracentre hole transitions in Be-doped bulk GaAs lie in this spectral range [阿. We believe that the emission of the GaAs/AlGaAs QW structure in this spectral range is connected with Be impurity contained in the semi-insulating GaAs substrate. The thickness of QW layers is not too high, so the exciting laser radiation can penetrate in the substrate and produce intracentre optical transitions in Be impurities according to the mechanism described for donors in the QW.

The part of the QW emission spectrum corresponding to low photon energies is shown more elaborately in Fig. 2.

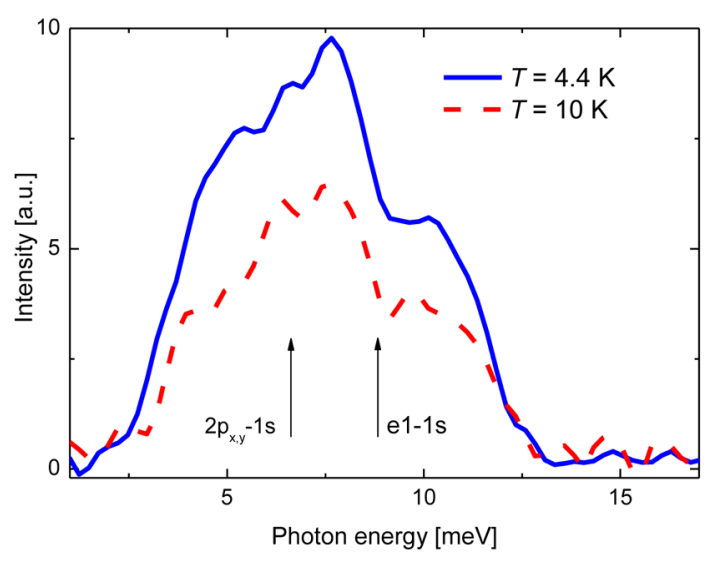

Fig. 2. Long wavelength part of the QW emission spectrum measured at two temperatures. Energies of some allowed electron transitions are shown with arrows.

We used energies of electron transitions calculated in [4] for the structure with the same parameters. Energies of electron transitions between the first subband and impurity ground state $e l \rightarrow 1 \mathrm{~s}$ and between excited and impurity ground states $2 \mathrm{p}_{x, y} \rightarrow 1 \mathrm{~s}$ are 8.8 and $6.5 \mathrm{meV}$, respectively, and are shown by arrows in Fig. 2. So, we can conclude that the spectrum shown in Fig. 2 is connected to electron transitions involving the impurity states.

In support of the suggested mechanism of terahertz radiation one can compare obtained emission spectra and spectra of photoconductivity measured in this structure before 刨. Both these spectra are shown in Fig. B. They have only slight differences. The photoconductivity spectrum has a short wavelength tail related to absorption due to transitions of electrons with high momentum to the states of the first subband. A high wavelength tail of emission spectrum can be related to electron transitions from $e 1$ subband to the highest excited states of impurity.

Our data correlatewell with the emission spectra measured in a similar structure under impurity breakdown 


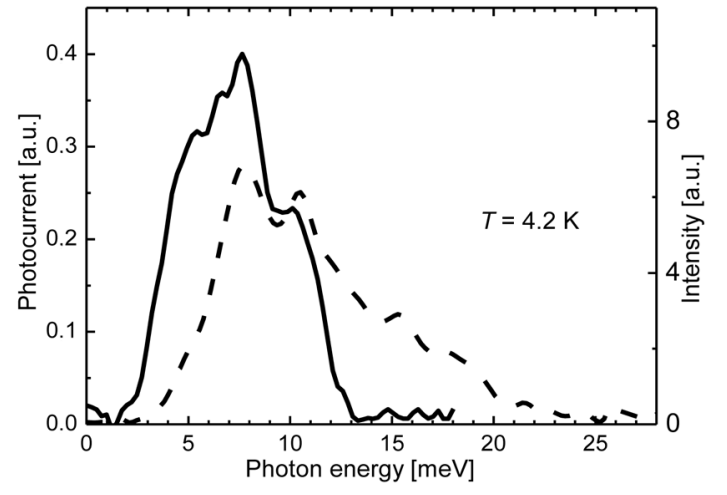

Fig. 3. Comparison between emission spectra (solid line) and spectra of photoconductivity (dashed line) measured in the same QW structure.

in the lateral electric field [4]. Emission spectra measured at these conditions also contain features in the 5-9 meV spectral range.

\section{THz emission at electrical excitation}

The second part of this work is devoted to the study of $\mathrm{THz}$ emission from doped QW nanostructures under electrical impurity breakdown in the lateral electric field. Our interest was concentrated on QW nanostructures doped with the Be acceptor. Beryllium has a high ionization energy of $28 \mathrm{meV}$ in bulk GaAs; therefore, in quantum wells ionization energy can exceed the energy of the optical phonon. As a result, intensity of the capture of non-equilibrium holes on the ground impurity level will be reduced and this level will remain comparatively empty. This situation will facilitate the optical hole transitions from the excited levels to the ground one.

We used MBE grown GaAs/AlGaAs structures consisting of 200 periods of $3.8 \mathrm{~nm}$ GaAs quantum wells separated with $7 \mathrm{~nm} \mathrm{Al}{ }_{0.4} \mathrm{Ga}_{0.6}$ As barriers. Quantum wells were doped in the $0.8 \mathrm{~nm}$ central region with the surface Be concentration of $3.2 \cdot 10^{11} \mathrm{~cm}^{-2}$.

Emission spectra were obtained using the technique described in the previous section. The electric field was applied in the form of a series of pulse trains. Train repetition was $87 \mathrm{~Hz}$, each train consisted of four $20 \mu$ s pulses.

The energy spectrum of Be acceptors in the GaAs/ AlGaAs QW structure was calculated in the envelope function approximation. Details of calculations are described in [6]. The acceptor Hamiltonian used was a $4 \times 4$ matrix operator including the Luttinger Hamiltonian, the QW confinement potential due to the valence band discontinuity, and the Coulomb potential. Results of the calculations are presented in Fig. 4.

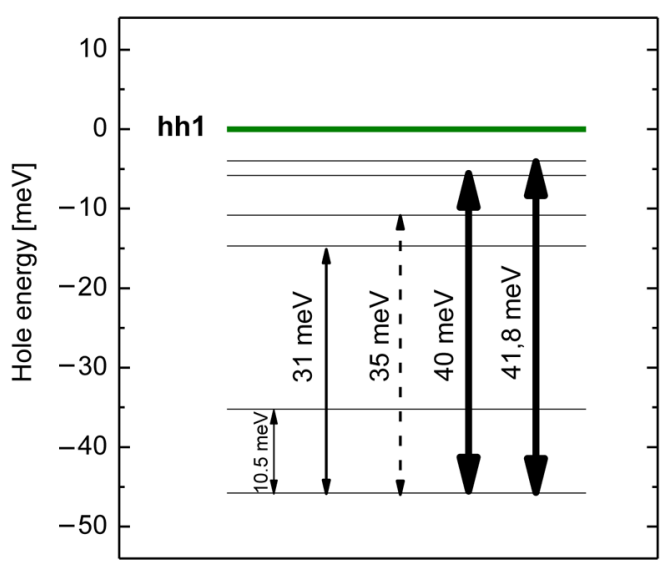

Fig. 4. The calculated acceptor levels bound to hh1 subband in the Be-doped GaAs/AlGaAs QW. Arrows 31-41.8 meV indicate allowed optical transitions (solid lines indicate s-polarization, dashed line indicates z-polarization). Wide arrows correspond to high value of the optical matrix element.

The current-voltage characteristic of the QW structure revealed a strong rise of the current starting with the electric field of approximately $500 \mathrm{~V} / \mathrm{cm}$ corresponding to impurity breakdown. Starting with this electric field depopulating the ground state of impurity we were able to observe terahertz emission. Experimentally observed terahertz emission spectra at the lateral electric field are shown in Fig. 5.

The observed spectra agree satisfactorily with results of the calculations shown in Fig. 4. We assume that the part of emission spectra in the range of 20 $28 \mathrm{meV}$ is related to optical transitions of hot holes from hh1 subband to acceptor states and to intracentre

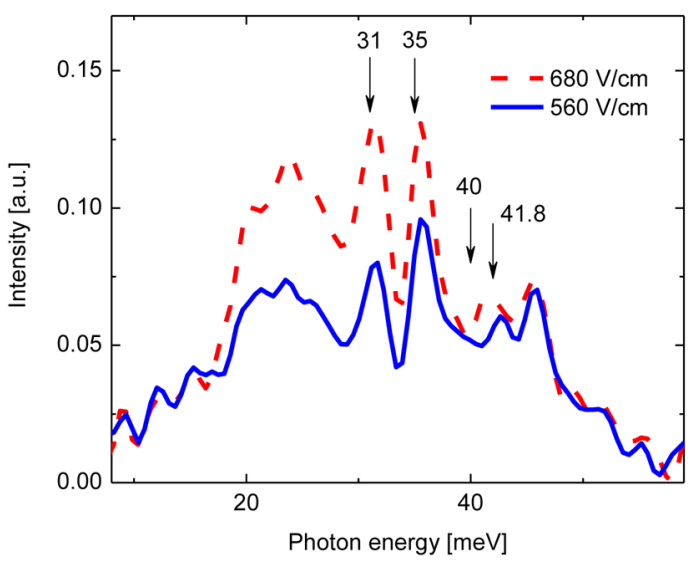

Fig. 5. Experimental emission spectra of Be-doped GaAs/AlGaAs QWs measured at $T=15 \mathrm{~K}$ under electrical excitation at different lateral electric field. Arrow marks show photon energies corresponding to Fig. 4. 
optical transitions between states of the acceptor. Emission peaks corresponding to hole transitions at 40 and $41.8 \mathrm{meV}$ are comparatively weak despite high values of the optical matrix elements because these energies belong to the restrahlen band.

\section{Acknowledgements}

The reported study was partially supported by the RFBR, research projects No. 12-02-01155 a, 1202-31034 mol_a, by the Russian Federal Program "Kadry" for 2009-2013, and the German-Russian Program of the Federal Ministry of Education and Research (BMBF).

\section{References}

[1] P.-C. Lv, R.T. Troeger, T.N. Adam, S. Kim, J. Kolodzey, I.N. Yassievich, M.A. Odnoblyudov, and M.S. Kagan, Appl. Phys. Lett. 85(1), 22-24 (2004), http://dx.doi.org/10.1063/1.1769589

[2] S.G. Pavlov, R.Kh. Zhukavin, E.E. Orlova, V.N. Shastin, A.V. Kirsanov, H.-W. Hübers, K. Auen, and
H. Riemann, Phys. Rev. Lett. 84(22) 5220-5223 (2000), http://dx.doi.org/10.1103/ PhysRevLett.84.5220

[3] A.V. Andrianov, A.O. Zakhar'in, Yu.L. Ivanov, and M.S. Kipa, JETP Lett. 91(2), 96-99 (2010), http:// dx.doi.org/10.1134/S0021364010020098

[4] D.A. Firsov, V.A. Shalygin, V.Yu. Panevin, G.A. Melentyev, A.N. Sofronov, L.E. Vorobjev, A.V. Andrianov, A.O. Zakhar'in, V.S. Mikhrin, A.P. Vasilev, A.E. Zhukov, L.V. Gavrilenko, V.I. Gavrilenko, A.V. Antonov, and V.Ya. Aleshkin, Semiconductors 44(11), 1394-1397 (2010), http://dx.doi. org/10.1134/S1063782610110023

[5] R.A. Lewis, T.S. Cheng, M. Henini, and J.M. Chamberlain, Phys. Rev. B 53(19), 12829-12834 (1996), http://dx.doi.org/ 10.1103/PhysRevB.53.12829

[6] V.A. Shalygin, L.E. Vorobjev, D.A. Firsov, V.Yu. Panevin, A.N. Sofronov, A.V. Andrianov, A.O. Zakhar'in, A.Yu. Egorov, A.G. Gladyshev, O.V. Bondarenko, V.M. Ustinov, N.N. Zinov'ev, and D.V. Kozlov, Appl. Phys. Lett. 90(16), 161128(1-3) (2007), http://dx.doi.org/10.1063/1.2730745

\title{
PRIEMAIŠŲ NULEMTA TERAHERCINĖ EMISIJA IŠ KVANTINIŲ ŠULINIŲ NANODARINIŲ
}

\author{
D.A. Firsov ${ }^{\text {a }}$, L.E. Vorobjev a, V.Yu. Panevin a , A.N. Sofronov ${ }^{\text {a }}$, R.M. Balagula a, \\ D.V. Kozlov ${ }^{b}$ \\ a Sankt Peterburgo valstybinis politechnikos universitetas, Sankt Peterburgas, Rusija \\ ${ }^{\mathrm{b}}$ Rusijos mokslu akademijos Fizikos mikrostruktūros institutas, Nižnij Novgorodas, Rusija
}

